\title{
Article Type: Review Article \\ Interdisciplinary periodontics: the multidisciplinary approach to the planning and treatment of complex cases
}

Authors

Karl Lyons

BDS, MDS, PhD, FRACDS

Professor and Chair in Restorative Dentistry

Department of Oral Rehabilitation and the Sir John Walsh Research Institute

Faculty of Dentistry

University of Otago

Dunedin, New Zealand

Ivan Darby

BDS, PhD, FRACDS(Perio)

Professor of Periodontics

Melbourne Dental School

The University of Melbourne

Melbourne, Australia

Abstract

Periodontics cannot be practiced in isolation and frequently many patients have multiple dental needs or medical health issues requiring management. In addition, pathology may manifest in the periodontal tissues and the onset and progression of periodontitis can be affected by systemic conditions such as diabetes and vice versa. The focus of this volume is interdisciplinary This is the author manuscript accepted for publication and has undergone full peer review but has not been through the copyediting, typesetting, pagination and proofreading process, which may lead to differences between this version and the Version of Record. Please cite this article as doi: 10.1111/prd.12187

This article is protected by copyright. All rights reserved 
periodontics, and the articles include the interactions and the interelationship between the periodontal tissues and diseases and endodontics, fixed prosthodontics, implant dentistry, aesthetics, gerodontology, radiology, orthodontics, paediatric dentistry, oral and maxillofacial surgery, oral pathology, special needs dentistry and general medicine. Previous volumes of Periodontology 2000 have covered some of the interactions with other dental disciplines, especially implant dentistry and through the periodontal/systemic interaction, but there has not been an volume on interdisciplinary periodontics. The intention is to show how and why periodontics should be interdisciplinary as well as the benefits of an interdisciplinary approach and some of the consequences if a discipline works in isolation.

The focus of this issue is interdisciplinary periodontics and the articles cover the interactions and interelationship between the periodontal tissues and diseases and endodontics, fixed prosthodontics, implant dentistry, aesthetics, gerodontology, radiology, orthodontics, paediatric dentistry, oral and maxillofacial surgery, oral pathology, special needs dentistry and general medicine. Periodontics isn't practiced in isolation and frequently many patients have multiple dental needs or medical health issues that require management. In addition, pathology may manifest in the periodontal tissues and the onset and progression of periodontitis can be affected by systemic conditions such as diabetes and vice versa. Previous volumes of Periodontology 2000 have covered some of the interactions with other dental disciplines, especially implant dentistry and through the periodontal/systemic interaction, but there has not been an volume that has just looked at interdisciplinary periodontics.

Understanding the interaction between endodontics and periodontics is of crucial importance to the clinician since endodontic-periodontal diseases often present challenges in assessment of diagnosis, treatment and prognosis (8). Etiologic factors such as microorganisms as well as contributing factors including trauma, root resorptions, perforations, cracks, and dental malformations play an important role in the development and progression of 
such lesions. Despite this, treatment and prognosis of endodontic-periodontal lesions vary and depend on the etiology, pathogenesis and a correct diagnosis of each specific condition. This chapter titled "Interaction between endodontics and periodontics" appraises the interrelationship between endodontic and periodontal lesions and provides biological and clinical evidence for diagnosis, prognosis and decision-making in the treatment of these conditions.

Although periodontal factors do not usually have a direct effect on the survival of a fixed prosthesis, harmony between the prosthesis and the periodontium is critical otherwise aesthetics, the longevity of the prosthesis and the periodontium will be compromised (1). In this chapter titled "Interdisciplinary interface between fixed prosthodontics and periodontics." the close interdisciplinary relationship between periodontics and prosthodontics is shown to avoid an unsatisfactory treatment outcome, requiring extensive and expensive re-treatment. The design of the prosthesis, the number and quality of the abutment teeth, the preparation and the pontic, the occlusion and material choice needs to be considered when planning prosthodontic treatment. The location of the preparation margin and the contour and emergence profile of the prosthesis will influence the response of the gingival tissues to the prosthesis. Pontic design and cleansibility also contribute to the response of the gingival tissues as well as the clinical and aesthetic outcome. Even an ideal pontic design will not prevent inflammation of the mucosa adjacent to the pontic if pontic hygiene is not maintained by removal of plaque. Case selection and the patients ability to be able to carry out adequate oral hygiene are therefore essential for longevity of the prosthesis, and regular reviews provide an opportunity for early detection and management of failures.

Dental implants are regularly placed in patients with a history of periodontitis, even though peri-implant tissues are susceptible to the same host-modulated plaque-induced factors that initiate and sustain periodontitis (11). In this chapter titled "Chronic periodontitis and implant dentistry" the evidence relating to a history of periodontitis as a risk factor for implant success and 
survival, and the role of supportive periodontal therapy in maintaining implants for individuals with a history of periodontitis is discussed.

Aesthetic considerations are a significant contributing factor in the management of prosthodontic cases and an interdisciplinary approach is often necessary to achieve an optimal result (3). The visible soft tissue architecture plays a key role in developing an aesthetic smile. Furthermore, an understanding of the relationship between the restorative margin and the gingiva is important for long-term stability of the result. The gingival architecture and health is of importance to ensure ideal aesthetics for prosthodontic work and close attention to both soft and hard tissues around the teeth before, during, and after restorative procedures will greatly improve the likelihood of a successful outcome. Moreover, knowing the options available in periodontal plastic surgery is important in aesthetic dentistry today. The relevant literature related to restorative and surgical procedures when performing aesthetic dentistry are reviewed in the chapter titled "The periodontal restorative interface: aesthetic considerations" and different approaches are illustrated with cases to explain the treatment approach that was utilized to improve the aesthetic outcome.

Periodontal conditions in older individuals and the available scientific data on periodontal treatment outcomes in individuals $\geq 75$ years of age are reviewed in the next chapter titled "Dental geriatrics and periodontitis" (7). The population of older people is increasing but the data on periodontal therapies and their efficacy is limited. Furthermore, although life expectancy has increased, this does not suggest that older people are medically healthy as the prevalence of most chronic diseases increases with age. Several chronic systemic diseases have been associated with periodontitis. The most frequent significant correlates with successful ageing include non-smoking, absence of disability, arthritis, and diabetes mellitus, however, periodontal diseases in older individuals may have specific features not consistent with periodontal diseases. Diagnostic criteria used for the assessment and diagnosis of periodontitis in younger adults may not be fully applicable in older individuals and the progression of periodontitis may be slower in older individuals. Older individuals with periodontitis may 
therefore benefit from more conservative treatment approaches. In older individuals declining health, perception of treatment needs, dietary changes, comorbidity with other diseases, and immune senescence are challenging factors in terms of therapies and understanding of periodontitis etiology

Contemporary periodontal therapy has evolved to become more interdisciplinary and increasingly involves complex treatments including bone and soft tissue regenerative procedures (10). Therapeutic options frequently require an imaging modality capable of providing a diagnostic osseous baseline and facilitating quantification of smaller increments of bony change, both loss and additive that is comparable over time. In this chapter titled "The emerging role of maxillofacial radiology in the diagnosis and management of complex periodontal cases " it is shown that intraoral and panoramic radiography are the most commonly used modalities to identify the location, quantify the amount, determine the pattern of marginal alveolar bone loss and, determine response to therapy. Unfortunately these procedures produce planar, two-dimensional (2-D) images which are subject to inaccuracies associated with inadequate exposure, processing or kilovoltage, image distortion due to variations in projection geometry and intra-and inter-observer variability. Cone beam computed tomography (CBCT) imaging offers specific advantages for periodontal diagnosis in that three-dimensional (3-D) images of dental and marginal alveolar bone structures can be rendered with geometric accuracy in one procedure. While there is currently no evidence to support the routine use of CBCT imaging for routine 3 -D bone mapping, it can has been shown to be clinically efficacious in demonstrating localized defects. Such defects include furcation involvement, intra-bony vertical and buccal/lingual defects, and it is proposed that in these situations, limited field of view, high resolution protocols are indicated.

Reduced periodontal support is a common challenge that clinicians often face during the rehabilitation of compromised dentitions (2). The close and intricate relationship between the periodontal tissues and the processes of tooth movement suggest that adjunct orthodontic therapy may play an important role in overcoming these problems. On the other hand, excessive movement of teeth 
beyond the anatomical boundaries of the alveolar process is commonly believed to contribute to further destruction of the periodontal tissues. In this chapter titled "Effect of orthodontic treatment on the periodontal tissues" clinical effects of various orthodontic tooth movements on the surrounding periodontal soft tissues and alveolar bone are evaluated. Particular emphasis is placed on specific tooth movements, such as extrusion, intrusion, space closure, and arch expansion. Limitations of current research are also highlighted and discussed.

Oral health of children and adolescents mirrors their general health (6). Because oral health care is often delivered in isolation from general health care, oral signs and symptoms do not always alert practitioners to their significance beyond the mouth. An example of this is the association of a higher risk of dental caries and periodontal disease in children and adolescents with pre-diabetic conditions in those who are overweight or obese. Dentists and periodontists therefore need to consider the health conditions their patients may have to aid in diagnosis and alert the practitioner to oral conditions that may not resolve without general health care intervention also. In this chapter titled "Management of periodontal health in children: pediatric dentistry and periodontology interface " the more common oral conditions involving periodontal health in children and adolescents and their diagnosis are discussed. The potential associated health problems are also presented together with the role of the pediatric dentist and periodontist in the management of children entering adulthood so that they do so with healthy dentitions.

Surgical procedures of the oral cavity can be performed by a number of dental specialists and clinicians (12). Due to the limited number of surgical procedures that can be performed inside the oral cavity, the boundary lines between specialties may become less distinct and lead to a confusion for general dentists in terms of patient referrals. In the chapter titled "Surgical management in dentistry - the interdisciplinary relationship between periodontology and oral and maxillofacial surgery" what the two surgical dental specialties of periodontics and oral and maxillofacial surgeons have to offer, is discussed and 
the routine inclusion of both periodontists and oral and maxillofacial surgeons in the multidisciplinary management of dental implant patients is highlighted.

The normal anatomy and physiology of the periodontium is well known to dentists and periodontists, as are the effects on the periodontium of plaqueassociated bacterial infection (9). However, the gingivae are involved in many other local and systemic conditions and the chapter titled "The nexus between periodontics and oral pathology" discusses how the periodontist and oral pathologist can work together to ensure these lesions are diagnosed and managed in a timely manner. Some of the less common pathological conditions that may affect one or more of the components of the periodontium are also discussed according to the classification of diseases, to provide a framework to guide investigations and management when an abnormality is detected.

Individuals with special needs are at more risk of dental disease, including periodontal diseases, and special needs patients have a greater prevalence and incidence of periodontal diseases compared with the rest of the population (4). Genetic or medical conditions, and/or the use of prescription medication or recreational substances may further increase the risk for susceptibility to periodontal disease. The success of preventing or controlling periodontal diseases amongst this group of patients has not been established. Even those individuals who access regular and comprehensive dental care appear to develop periodontal diseases as they age at a rate comparable to the natural history of the disease. The reasons behind this lack of success of interventions in reducing the incidence of periodontal diseases are complex and may relate to the professional challenges in treating individuals with special needs.

In many countries, dental services, unlike other healthcare services, are not covered by the principle of universal access (5). It has only been in the last couple of decades that there has been a greater interaction between medicine and dentistry. Various health care systems around the world may provide public dental services to the young and the disadvantaged, but relatively few provide 
access to all. Public policy appears to not appreciate the vast economic, health and social implications that poor oral health has on the overall health of an individual. Recognizing and acting on the interrelatedness between oral health and overall health helps protect patients from pathological diseases such as infective endocarditis, suboptimal glycaemic control, and deterioration in renal function. In this chapter titled "The place of periodontal examination and referral in general medicine " some of the medical conditions that patients are predisposed to as a result of poor dental care are examined and case examples are provided to support this hypothesis. The importance of a strong relationship that is needed between the dentist and the physician, is shown and suggestions are offered for a multidisciplinary approach.

This volume aims to show how and why periodontics should be interdisciplinary and each of the chapters shows the benefits of an interdisciplinary approach and some of the consequences if a discipline works in isolation.

Acknowledgement

We are grateful for the significant contribution of Professor Gregory Seymour to this volume of Periodontology 2000.

\section{References}

1. Abduo J, Lyons K. Interdisciplinary interface between fixed prosthodontics and periodontics. Periodontol 2000 2017: 7?:

2. Antoun JS, Mei L, Gibbs K, Farella M. Effect of Orthodontic Treatment on the Periodontal Tissues. Periodontol 2000 2017: 7?:

3. Bennani V, Ibrahim H, Al Harthi L, Lyons K. The periodontal restorative interface: aesthetic considerations. Periodontol 2000 2017: 7?:

4. Brown L, Ford PJ, Symons AL. Periodontal disease and the special needs patient. Periodontol 2000 2017: 7?:

5. Chan S, Maurice AP, Pasternak GM, West MJ. The place of periodontal examination and referral in general medicine. Periodontol 2000 2017: 7?: 
6. Drummond BK, Brosnan MG, Leichter JW. Management of Periodontal Health in Children: Pediatric Dentistry and Periodontology Interface. Periodontol 2000 2017: 7?:

7. Persson RG. Dental Geriatrics and Periodontitis. Periodontol 2000 2017: 7?:

8. Rotstein I. Interaction between Endodontics and Periodontics. Periodontol 2000 2017: 7?:

9. Rich A, Seo B, Parachuru V, Hussaini HM. The Nexus between Periodontics and Oral Pathology. Periodontol 2000 2017: 7?:

10. Scarfe WC, Azevedo B, Pinheiro LR, Priaminiarti, Sales MAO. The emerging role of maxillofacial radiology in the diagnosis and management of complex periodontal cases. Periodontol 2000 2017: 7?:

11. Smith MM, Knight ET, Al-Harthi L, Leichter JW, Seymour GJ. Periodontal considerations in implant dentistry. Periodontol 2000 2017: 7?:

12. Tong DC. Surgical management in dentistry - the interdisciplinary relationship between periodontology and oral and maxillofacial surgery. Periodontol 2000 2017: 7?:

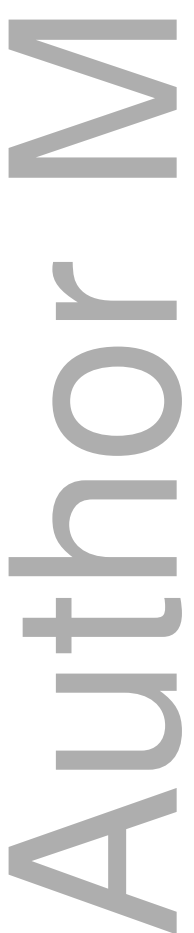




\section{University Library}

\section{- M M N E R VA A gateway to Melbourne's research publications}

Minerva Access is the Institutional Repository of The University of Melbourne

Author/s:

Lyons, KM;Darby, I

Title:

Interdisciplinary periodontics: the multidisciplinary approach to the planning and treatment of complex cases

Date:

2017-06-01

Citation:

Lyons, K. M. \& Darby, I. (2017). Interdisciplinary periodontics: the multidisciplinary approach to the planning and treatment of complex cases. PERIODONTOLOGY 2000, 74 (1), pp.7-10. https://doi.org/10.1111/prd.12187.

Persistent Link:

http://hdl.handle.net/11343/292792 